\title{
Índices de competitividad: Análisis de resultados para Colombia 2015-2016
}

\section{Competitiveness indices: Results analysis for Colombia Colombia 2015-2016}

\author{
págs. $150-164$ \\ Grupo de investigación 1: Prospectiva Urbano Territorial \\ Línea de investigación: Ciudades Competitivas \\ Grupo de investigación 2: Centro de Investigación en Competitividad Empresarial \\ Línea de investigación: Estrategias de Calidad y Competitividad \\ John Fredy Ramírez Ríos• Angélica María Alzate Ibañez••
}

Recibido: agosto 01 de 2016

\section{RESUMEN}

En la actualidad, los países en desarrollo han aumentado su preocupación por la competitividad nacional y su posición en los índices de desempeño competitivo internacional. Este trabajo presenta un primer análisis de los resultados que Colombia ha obtenido en las mediciones de competitividad en el 2015-2016, desde una perspectiva de desarrollo territorial. Se consideran las mediciones de los índices más conocidos: The Global Competitiveness Report, Doing Business, y The IMD World Competitiveness Yearbook Los resultados exponen un panorama estable a nivel macroeconómico y financiero, y de incertidumbre en ámbitos institucionales y territoriales.

Palabras clave: competitividad, evaluación comparativa, desempeño, índices de clasificación, desarrollo territorial
Aceptado: noviembre 25 de 2016
Key word: competitiveness, benchmarking, performan$\mathrm{ce}$, indices ranking, territorial developing

\footnotetext{
- Sociólogo. Magíster en Urbanismo. Docente. Facultad de Educación Permanente y Avanzada. Fundación Universidad de América. email: john.ramirez@investigadores.uamerica.edu.co

-• Ingeniera Química. Docente. Facultad de Educación Permanente y Avanzada. Fundación Universidad de América. email: angelica.alzate@investigadores.uamerica.edu.co
} 
LÍNEA DE INVESTIGACIÓN: ESTRATEGIAS DE CALIDAD Y COMPETITIVIDAD

\section{INTRODUCCIÓN}

La preocupación sobre la competitividad nacional en países en desarrollo ha aumentado considerablemente en los últimos años. A nivel mundial los países se clasifican según criterios y medidas de destreza competitiva nacional que conducen al cálculo de indicadores compuestos. Si bien su impacto real es difícil de evaluar, estos índices han sido objeto de intenso análisis, sobre todo en Asia Oriental, donde la competitividad se considera una cuestión de supervivencia económica nacional (Lall, S., 2001).

Recientemente Colombia ha empezado a participar en mediciones internacionales de competitividad nacional y urbana, cuyos índices se componen de indicadores macroeconómicos y financieros (Ramírez et al., 2007; Sobrino, 2005) basados en hard data: PIB, inflación, PIB per cápita, salario mínimo, etc; y de indicadores institucionales y territoriales (Tóth, 2014, p. 3) basados en soft data: niveles de transparencia de las entidades gubernamentales, niveles de ética pública, capital social, redes de infraestructura de ciencia y tecnología, etc.

Estos índices ofrecen un diagnóstico económico, social e institucional cada vez más completo de los países que se someten a sus mediciones. Ello le facilita a los hacedores de política pública generar o direccionar programas y políticas de desarrollo con enfoque no solo económico sino verdaderamente territorial, que permitan estimular ya sea el emprenderismo (Sousa, 2011), el autoempleo (Affuso \& Camagni, 2010), la innovación social y tecnológica (Méndez, 2002), la especialización flexible (Revilla, Schiller, \& Meyer, 2013), la descentralización administrativa a nivel regional, urbano y local (Lang, 2009) o la gobernanza responsable, (Benzaquen, del Carpio, Zegarra, \& Valdivia, 2010; Lira, 2005; Organisation for Economic Co-operation and Development - OECD, 2001; Ramírez, Osorio, \& Isidro, 2007).

En este artículo se describe brevemente cómo el discurso de la competitividad incluye actualmente no solo asuntos estrictamente económicos y financieros, sino también institucionales y territoriales; y el efecto que ello tiene en la composición de las dimensiones evaluadas por los índices internacionales de competitividad más importantes. Se realiza un análisis de los resultados que Colombia ha obtenido en esos índices.

\section{MÉTODO}

El diseño de la investigación es tipo descriptivo, según López Niño (2015) "la investigacion descriptiva consiste en caracterizar un hecho, fenómeno, individuo o grupo con el fin de establecer su estructura o comportamiento". Iniclamente, se realiza una reflexión teórica sobre evidencia empírica proveniente de fuentes secundarias. Se utilizan datos del servicio de indexación Scopus para demostrar cambios en los discursos de competitividad, por medio del análisis bibliométrico de la producción generada alrededor de ese concepto desde 1966, siguiendo la metodología descrita por Ramírez et. al. (2016). Finalmente, se revisaron los resultados para Colombia de tres índices internacionales de competitividad: The Global Competitiveness Report (2015-2016), Doing Business (2016), y The Imd World Competitiveness Yearbook (2016). 


\section{RESULTADOS}

De un énfasis economicista de la competitividad a una visión integral del desarrollo territorial.

El término de competitividad usualmente se asocia con la gestión económica y empresarial. Un análisis general de la producción científica que referencia el término desde 1966 hasta 1991, indica que éste apareció inicialmente en documentos que describían procesos propios de la biología, la medicina, la ecología, la psicología organizacional, la ingeniería industrial; y de manera secundaria en documentos relacionados con la administración y los negocios, la economía, el comercio internacional, la planeación urbana y las ciencias sociales, como se observa en la figura 1.

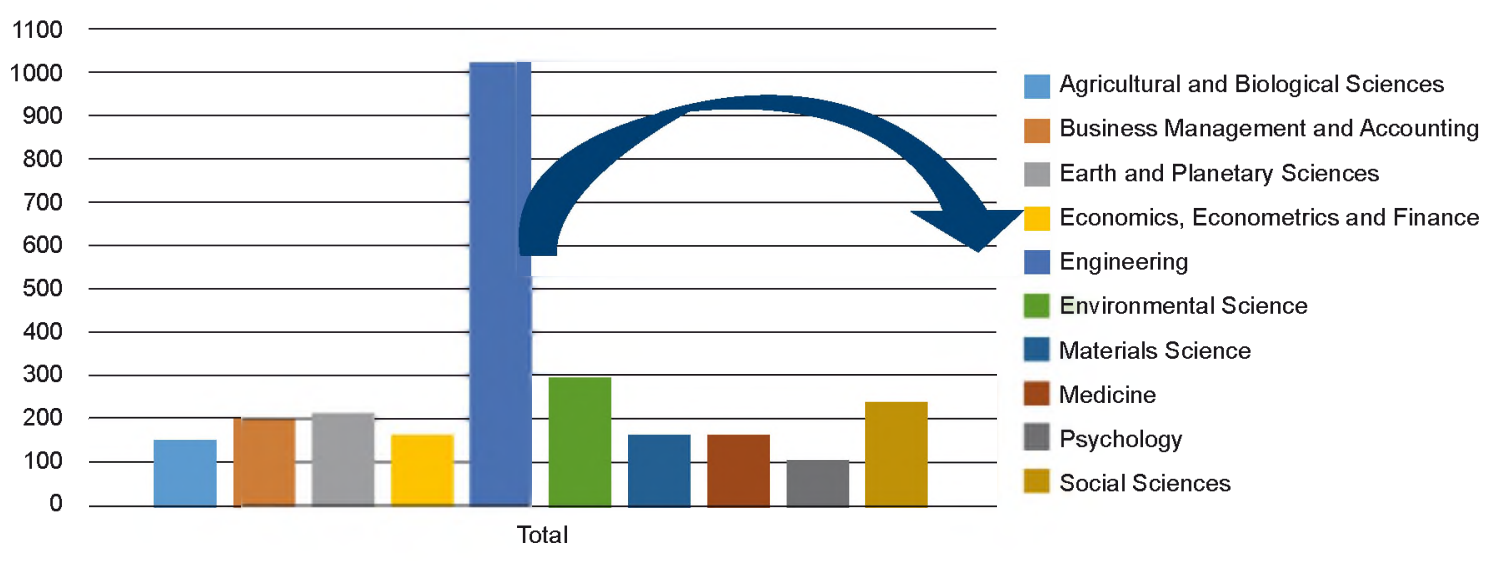

Figura 1. Uso del término competitividad en la literatura científica internacional 1966-1991

Fuente: Elaboración propia con base en datos provistos por Scopus. Copyright (c) 2016 Elsevier B.V. All rights reserved.

A partir de 1992, el término competitividad se posicionó plenamente en la producción científica de las áreas de la economía, la administración y los negocios; como una temática de estudio independiente (Ver figura 2). Ese posicionamiento fue acompañado de un cuestionamiento a las políticas de sustitución de importaciones, y de un impulso al comercio internacional en un contexto de economía globalizada (Buckley, Pass, \& Prescott, 1988; Porter, 1990). Un hecho que evidencia la asociación descrita entre comercio internacional y competitividad, se puede encontrar en el Report from the Select Committee of the House of Lords on Overseas Trade (1985). Según Brucker, Pass \& Prescott (1988), este reporte contiene la primera definición canónica de competitividad desde una perspectiva netamente económica, expuesta de la siguiente manera:

A firm is competitive if it can produce products and services of superior quality and lower costs than its domestic and international competitors. Competitiveness is synonymous with a firm's longrun profit performance and its ability to compensate its employees and provide superior returns to its owners (p.176).

Desde el punto de vista de la administración y negocios, la competitividad se refería exclusivamente a la capacidad de las empresas y de las naciones de aumentar sus márgenes de productividad en un contexto de competencia internacional. En esa medida, los indicadores que se usaban para valorar el desempeño competitivo, medían aquellas variables pertinentes de índole macro y micro económicas, y de economías de escala. 
LÍNEA DE INVESTIGACIÓN: ESTRATEGIAS DE CALIDAD Y COMPETITIVIDAD

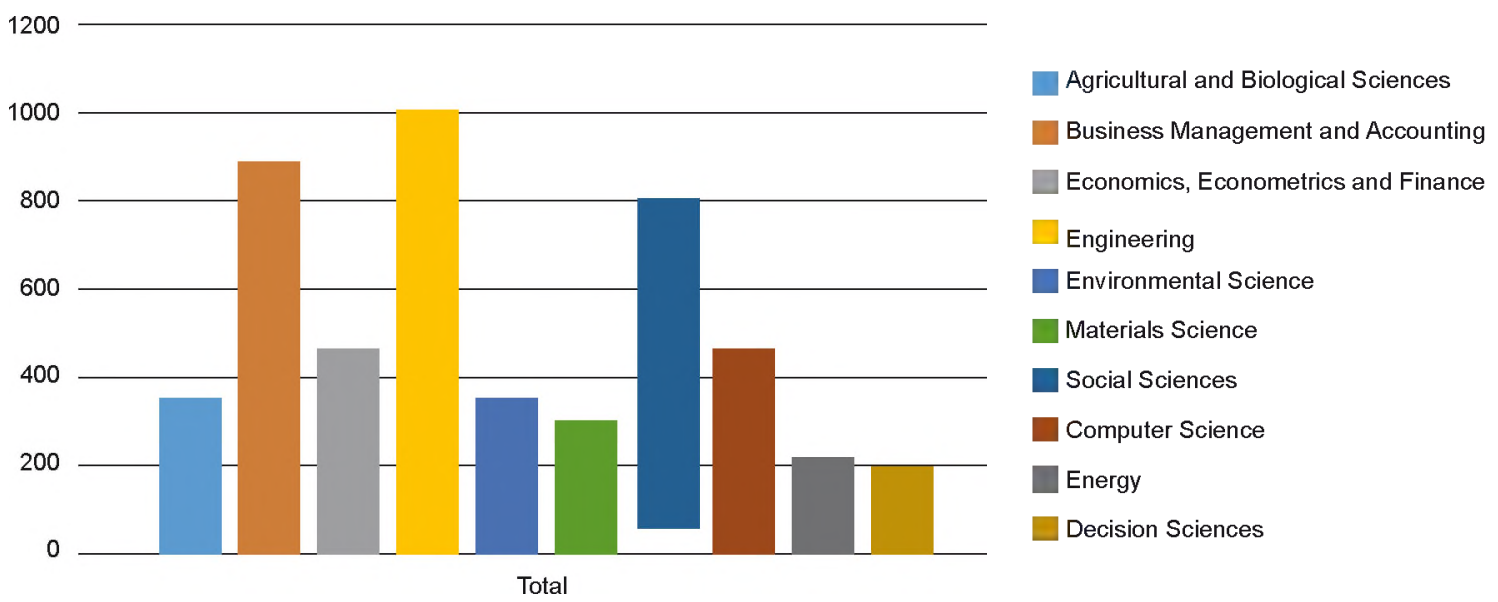

Figura 2. Uso del término competitividad en la literatura científica internacional 1992-2015

Fuente: Elaboración propia con base en datos provistos por Scopus.Copyright (C) 2016 Elsevier B.V. All rights reserved.

Sin embargo, a finales de la década de los noventa el panorama presenta un cambio radical. El concepto de competitividad incorporó nuevas aproximaciones al comercio internacional, desde una perspectiva cercana a las clásicas teorías de la localización (Tóth, 2014, p. 4) en la naciente disciplina de la geografía económica (Krugman, 1995; M. E. Porter, 2000). Este enfoque ha generado un efecto importante en las mediciones recientes de competitividad, las cuales han empezado a incorporar no solamente indicadores de desempeño de variables macro y micro económicas; sino también de otro tipo de variables propias de las economías de aglomeración y del desarrollo territorial. En otras palabras, la competitividad dejó de ser un asunto interesado en el desempeño exclusivamente de las economías nacionales o de las unidades económicas, sino además de las sociedades y los espacios físicos en las cuales éstas operan.

De esta manera, los resultados de aplicar los indicadores de competitividad contemporáneos no solo son útiles para el direccionamiento de políticas económicas nacionales, industriales o empresariales; sino también para el desarrollo de estrategias espaciales orientadas a la oferta de recursos territoriales (Camagni, Caragliu, \& Perucca, 2011). Los índices actuales de competitividad evalúan también el territorio entendiéndolo como una organización y/o mercancía que se puede inventariar, modelizar e intervenir en todas aquellas dimensiones que le sean cuantificables; con el fin de que éste se haga más competitivo, como también a las empresas que se asientan sobre él.

Así pues, estas nuevas aproximaciones a la competitividad que hoy dominan conceptualmente los documentos que contienen índices y rankings, se basan en dos principios fundamentales: primero, que el territorio puede ser concebido como un capital: un asset con propiedades similares al de otros capitales físicos, financieros, sociales, o intelectuales, etc; segundo, que como todo capital, puede ser objeto de planificación y gestión que lo hagan más productivo y eficiente¹.

1 La historia detrás de los principios de la nueva economía del comercio internacional debe ser objeto de otro estudio. Para efectos de este estudio se considerará que: "regions are not granted some productive specialization and some role within the spatial division of labour whenever they prove less competitive in all production sectors with respect to the external territories... unlike countries, regions are not subject to the principle of "comparative advantage" governing international specialization and trade, attributing each partner country some specialization sectors and a condition of full employment" (Camagni, 1992). (Organisation for Economic Co-operation and Development - OECD, 2001, p. 151) 
En la Tabla 1 se resumen algunas definiciones paradigmáticas de capital territorial y que permiten las mediciones contemporáneas cuantitativas y cualitativas de la competitividad, y las cuales son sintetizadas por Tóth (2014).

Tabla 1.

Definiciones paradigmáticas de capital territorial según Tóth (2014)

\begin{tabular}{|c|c|}
\hline Autor & Definición \\
\hline Camagni, 2009 & $\begin{array}{l}\text { "a set of localized assets-natural, artificial, human, organizational, relational } \\
\text { and cognitive-that comprise the potential of a certain territory" (p.1) }\end{array}$ \\
\hline Van der Ploeg et al., 2008 & $\begin{array}{l}\text { "the amount and intertwinement of different forms of capital (or different } \\
\text { resources) entailed in, mobilized and actively used in (and reproduced by) the } \\
\text { regional economy and society" (Van der Ploeg et al., 2008, p. 13) }\end{array}$ \\
\hline Ventura et al., 2008 & $\begin{array}{l}\text { "a stock of resources specific to the place and available to those who live and } \\
\text { work in the territory. These resources (material and immaterial) are common } \\
\text { goods for a local community" (p. 160) }\end{array}$ \\
\hline Giaime, 2011 & $\begin{array}{l}\text { "the whole of the local assets which if adequately mobilized provide } \\
\text { comparative advantages to those who live and work there" (p. 9) }\end{array}$ \\
\hline
\end{tabular}

El principio de que el territorio pueda ser tratado como un capital, o una organización que aglutina capitales, si bien es admitido con relativo consenso político y académico; resulta de difícil cuantificación y representación en índices de competitividad. Aún es motivo de amplio debate la robustez metodológica y el alcance de éstos, dada la variedad de indicadores que deben ser construidos, de las especificidades de las variables cuantitativas y cualitativas que ellos miden (macro-económicas, financieras, de desarrollo empresarial y territorial), y de los pesos ponderados que deben tener dentro de los índices que los conglomeran. Al respecto Tóth (2014) afirma que:

Obviously, a widespread agreement on the instruments that can be used to measure territorial capital still does not exist. There is no consensus on which set of variables or proxies should be involved in territorial capital analyses; to this time little effort has been made to find the best group of indicators or individual proxies. There is still a need for finding the best variables; however, on the one hand, every work offered a unique example of how great the importance of local values is; on the other, each empirical investigation may help correct and develop better understanding relating to the precise measurement of territorial capital in the future (p. 10).

No obstante las consideraciones anteriores, hoy existen índices de competitividad estandarizados que combinan indicadores de variables macroeconómicas y territoriales. De acuerdo con Buckler, Pass \& Prescott (1988) éstos índices pueden medir la competitividad, independientemente de las escalas territoriales, desde tres perspectivas: como resultado, como potencialidad y como proceso, de acuerdo a la siguiente descripción y como se ilustra en la Figura 3.

- como un resultado (performance), es decir, concebir la competitividad como una suma lineal de factores. Normalmente, los datos que son resultados de estadísticas oficiales (PIB, PIB per cápita, Inflación, etc) sirven para construir indicadores de este tipo de perspectiva de medición basado fundamentalmente en hard data. 
LÍNEA DE INVESTIGACIÓN: ESTRATEGIAS DE CALIDAD Y COMPETITIVIDAD

- como una potencialidad, es decir, concebir la competitividad como un escenario deseable. Ejemplo de esta perspectiva es la presencia de clústeres económicos, de economías de aglomeración, de buffers de influencia de economías regionales, etc.

- como un proceso, es decir, concebir la competitividad como una forma de gestión socio-económica. Esta perspectiva explica la presencia reciente de indicadores de institucionalidad y desarrollo territorial, como por ejemplo, la percepción que tienen los empresarios de niveles de corrupción y/o transparencia en las instituciones de gobierno, la facilidad que perciben para hacer negocios, el peso que tienen los conflictos y la violencia interna en los intentos de emprenderismo empresarial etc ${ }^{2}$. Ios resultados de la aplicación de encuestas (tipo DELPHI) o de otros instrumentos (FODA, estudios de prospectiva, o de econometría espacial) sirven para construir indicadores de potencialidad o de proceso en el desarrollo territorial (soft data) (Buckley et al., 1988).

En general, los índices actuales de competitividad combinan esas tres formas de aproximación a la medición de competitividad, con diferencias en los indicadores que toman en cuenta y en los modelos matemáticos de agregación de sus valores. No obstante esas diferencias, todos ellos ofrecen evidencia empírica ya sea del desempeño económico competitivo de un país, región o ciudad; pero también de las potencialidades o procesos competitivos de dichas escalas desde el desarrollo territorial.

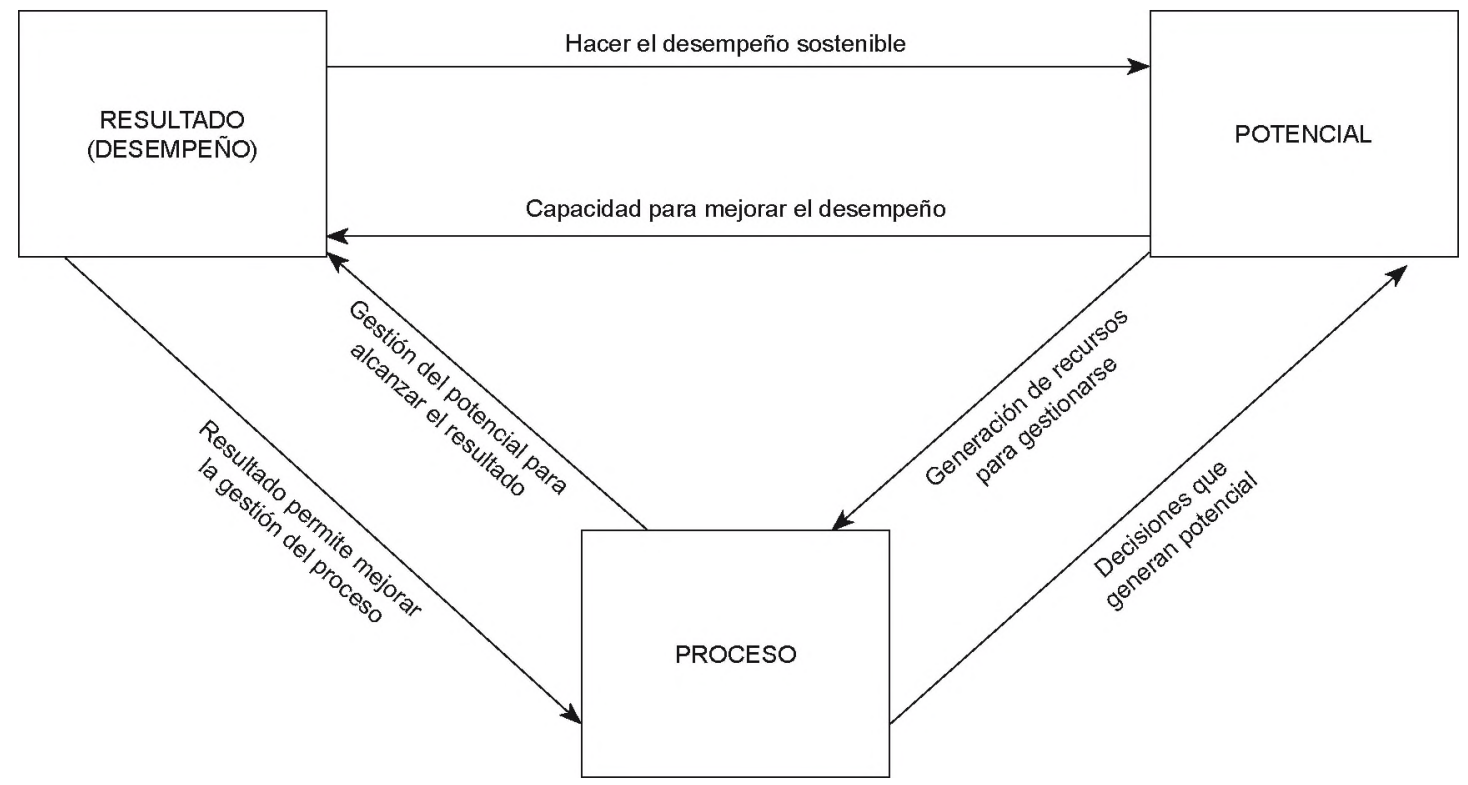

Figura 3. Enfoques de Medición de la Competitividad

Fuente: Adaptado de Buckley et al., 1988, p. 78.

2 De acuerdo con Sobrino (2005) se podría afirmar que la mayoría de los indicadores de los índices de competitividad global y urbana funcionan actualmente como medidas cuantitativas del éxito o fracaso de un país o ciudad para ser competitiva, sin pretensión de explicar tal desempeño" (p. 129). Haría falta, entonces, indicadores que midan los "determinantes de la competitividad" (Sobrino, 2005, p. 146), como lo son los que apuntan a la generación de evidencias de las dinámicas territoriales que condicionan el tipo de resultados en competitividad. 
En la Tabla 2 se desagregan por componentes temáticos los índices más importantes de competitividad tanto a escala nacional, regional y urbana. Sus indicadores se pueden agrupar en dos grandes componentes: aquellos que miden el desempeño competitivo, potencialidades o procesos de variables macro-económicas, financieras y empresariales; y por otra parte aquellos que miden el desempeño competitivo, potencialidades o procesos del desarrollo territorial, fundamentalmente en términos de infraestructura, instituciones y gobierno. El peso ponderado de este grupo de indicadores asociados a variables territoriales generalmente es menor para el cálculo del valor del índice total, sin embargo, han adquirido un mayor peso como aspectos determinantes para la competitividad nacional o urbana.

Tabla 2.

Componentes temáticos de los índices globales más importantes de competitividad

\begin{tabular}{|c|c|c|}
\hline \multirow[b]{2}{*}{ INDICE Y REPORTE } & \multicolumn{2}{|l|}{ COMPONENTE TEMÁTICO } \\
\hline & $\begin{array}{l}\text { Asociado al } \\
\text { Desarrollo territorial }\end{array}$ & $\begin{array}{l}\text { Asociado a la Economía, las } \\
\text { finanzas y las empresas. }\end{array}$ \\
\hline $\begin{array}{l}\text { The IMD World } \\
\text { Competitiveness } \\
\text { Scoreboard }\end{array}$ & $\begin{array}{l}\text { Infraestructura } \\
\text { Eficiencia del gobierno }\end{array}$ & Desempeño económico \\
\hline $\begin{array}{l}\text { En The IMD World } \\
\text { Competitiveness Yearbook }\end{array}$ & Eficiencia de los negocios & \\
\hline $\begin{array}{l}\text { The Global } \\
\text { Competitiveness Index } \\
\text { En } \\
\text { The Global } \\
\text { Competitiveness Report }\end{array}$ & $\begin{array}{l}\text { Instituciones } \\
\text { Infraestructura } \\
\text { Salud y educación primaria } \\
\text { Educación superior y Formación técnica } \\
\text { Innovación } \\
\text { Eficiencia del mercado laboral } \\
\text { Preparación tecnológica } \\
\text { Sofisticación de los negocios }\end{array}$ & $\begin{array}{l}\text { Ambiente macroeconómico } \\
\text { Eficiencia del mercado de bienes } \\
\text { Tamaño del mercado } \\
\text { Desarrollo del mercado financiero }\end{array}$ \\
\hline $\begin{array}{l}\text { Distance to frontier (DTF) } \\
\text { Score } \\
\text { En: Doing Business }\end{array}$ & $\begin{array}{l}\text { Manejo de permisos de construcción } \\
\text { Obtención de electricidad } \\
\text { Cumplimiento de contratos }\end{array}$ & $\begin{array}{l}\text { Apertura de un negocio } \\
\text { Registro de propiedades } \\
\text { Protección de los inversores } \\
\text { Obtención de crédito } \\
\text { Pago de impuestos } \\
\text { Comercio transfronterizo } \\
\text { Resolución de la insolvencia }\end{array}$ \\
\hline
\end{tabular}


LÍNEA DE INVESTIGACIÓN: ESTRATEGIAS DE CALIDAD Y COMPETITIVIDAD

\begin{tabular}{lll}
\hline \multirow{2}{*}{ INDICE Y REPORTE } & COMPONENTE TEMÁTICO & \\
\cline { 2 - 3 } & $\begin{array}{l}\text { Asociado al } \\
\text { Desarrollo territorial }\end{array}$ & $\begin{array}{l}\text { Asociado a la Economía, las } \\
\text { finanzas y las empresas. }\end{array}$ \\
\hline $\begin{array}{l}\text { Global Urban } \\
\text { Competitiveness Report } \\
\text { (2011-2012) }\end{array}$ & $\begin{array}{l}\text { Sistema de indicadores de } \\
\text { competitividad global de los factores }\end{array}$ & $\begin{array}{l}\text { Sistema de indicadores de la } \\
\text { competitividad global de la } \\
\text { producción }\end{array}$ \\
the financial tsunami & $\begin{array}{l}\text { Necesidades locales } \\
\text { Infraestructura }\end{array}$ & $\begin{array}{l}\text { Ambiente Interior } \\
\text { Sistema público } \\
\text { Conexión global }\end{array}$ \\
$\begin{array}{l}\text { Efectividad institucional } \\
\text { Hot Spots Benchmarking } \\
\text { Global City } \\
\text { Competitiveness (2012) }\end{array}$ & $\begin{array}{l}\text { Fama social y cultural Atractividad global } \\
\text { Ambiente y amenazas naturales }\end{array}$ & $\begin{array}{l}\text { Sistema de indicadores de la } \\
\text { competitividad global de la industria } \\
\text { Madurez financiera }\end{array}$ \\
\hline
\end{tabular}

\section{Resultado de mediciones de competitividad para Colombia}

Colombia se posiciona actualmente en tres índices internacionales de competitividad nacional. En la Tabla 3 se muestran los resultados con más bajos valores desagregados por indicador que el país obtiene, la mayoría vinculados al componente temático asociado al desarrollo territorial.

Tabla 3.

Resultados de Colombia por indicadores desagregados de índices internacionales de competitividad en relación al desarrollo territorial.

THE GLOBAL COMPETITIVENESS REPORT (2015-2016)

\begin{tabular}{lcc}
\hline \multicolumn{1}{c}{ Indicador $^{1}$} & $\begin{array}{c}\text { Valor } \\
\mathbf{1 - 7} \text { (Mejor) }\end{array}$ & $\begin{array}{c}\text { Posición entre } \mathbf{1 4 0} \\
\text { Países }\end{array}$ \\
\hline Quality of railroad infrastructure & 1.4 & 106 \\
Public trust in politicians & 1.8 & 131 \\
Diversion of public funds & 2.2 & 131 \\
Quality of roads & 2.7 & 126 \\
Favoritism in decisions of government officials & 2.4 & 115 \\
Venture capital availability & 2.7 & 70 \\
Wastefulness of government spending & 2.6 & 100 \\
Burden of government regulation & 2.6 & 126 \\
Business costs of terrorism & 2.9 & 132 \\
\hline
\end{tabular}




\begin{tabular}{llc}
\hline Judicial independence & 2.7 & 114 \\
Effect of taxation on incentives to invest & 2.7 & 131 \\
Business costs of crime and violence & 2.7 & 132 \\
Organized crime & 2.8 & 135 \\
Ease of access to loans & 2.8 & 77 \\
Company spending on R\&D & 2.9 & 96 \\
\hline
\end{tabular}

DOING BUSINESS (2016)

Agregados de indicadores

Valor
$0-100$ (Mejor)

Posición entre 189

Países

$\begin{array}{llr}\text { Cumplimiento de Contratos }^{2} & 29.66 & 180 \\ \text { Comercio transfronterizo } & 62.83 & 110 \\ \text { Pago de Impuestos } & 63.32 & 136\end{array}$

THE IMD WORLD COMPETITIVENESS YEARBOOK (2016)

Agregados de indicadores

Posición entre 60

Países

Societal Framework

59

Scientific Infrastructure

57

International Trade

56

Institutional Framework

51

1 Solo se referencian los indicadores que asumen un valor entre 0.0 y 7.0. Se seleccionaron los que se ubicaron por debajo del valor 3.0 (no inclusive).

2 Los indicadores que lo componen son tres: tiempo en días para resolver una disputa, costo (porcentaje del valor de lo demandado), y número de procedimientos. Sorprende los resultados para Colombia en los dos primeros indicadores: una disputa comercial en Colombia se demora en resolver en promedio cuatro años, y representa alrededor del $50 \%$ de lo demandado (Banco Mundial, 2014).

En el ranking publicado en The Global Competitiveness Report 2015-2016 (2015) el pais obtuvo la posición 61 entre 148 países. La tendencia general ha sido positiva con respecto a la medición 2014-2015 cuando ocupó la posición 66. "The top Latin American performer is Chile (35th), followed by Panama (50th) and Costa Rica (52nd). Mexico and Colombia are rapidly approaching the top three after improving four and five positions, respectively" (World Economic Forum, 2015, p. 22)

Si bien el desempeño del país es sobresaliente en los indicadores macroeconómicos y financieros, con ligeras mejorías en los indicadores relacionados con salud y educación, en este ranking resulta evidente que el país mantiene un desempeño bajo en indicadores relacionados con instituciones e infraestructura, temáticamente asociados al desarrollo territorial (Figura 4), como lo destaca el mismo informe: "Other areas for improvement are the country's institutional framework, especially public institutions (125th), with corruption (126th) and security (134th) remaining dire. 
LÍNEA DE INVESTIGACIÓN: ESTRATEGIAS DE CALIDAD Y COMPETITIVIDAD

Structural reforms to foster competition (127th) and improve infrastructure, specifically the overall quality of transport (98th), would further enhance competitiveness" (World Economic Forum, 2015, p. 31)

De hecho, los resultados que Colombia obtuvo en los indicadores con ponderaciones iguales o inferiores a 3.0, corresponden mayoritariamente a los pilares instituciones e infraestructura. Cinco indicadores muestran una tendencia negativa de con respecto al informe 2013-2014, y dos indicadores desmejoraron a un valor menor a 3.0. Los valores de indicadores que no desmejoraron, simplemente se mantuvieron igual o tuvieron una mejoría insignificante de 0.100 .2 puntos. Solamente un valor de indicador superó la barrera de los 3 puntos.

Como complemento a los resultados de las mediciones de este índice se analizaron los resultados de la encuesta tipo Delphi publicados en World Economic Forum (2015). Los empresarios enfatizan sobre las deficiencias en las variables asociadas al desarrollo territorial, tendencias que impiden el salto definitivo que el país requiere en competitividad (Figura 5).

\section{GCI 2015-2016}

$\mathrm{GCl}$ 2014-2015 (out of 144)

GCl 2013-2014 (out of 148)

$\mathrm{GCl}$ 2012-2013 (out of 144)

Basic requirement $(40,0 \%)$

1st pillar: Institutions

2nd pillar: Infrastructure

3rd pillar: Macroeconomic environment

4th pillar: Health and primary education

Efficiency enhancers (50.0\%)

5th pillar: Higher education and training

6th pillar Goods market efficiency

7th pillar: Labor market efficiency

8th pillar: Financial market development

9th pillar: Technological readiness

10th pillar: Market size

Innovation and sophistication factors (10.0\%)

11th pillar: Business sophistication

12th pillar: Innovation

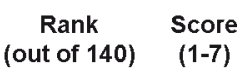

61

$66 \quad 4.2$

$69 \quad 4.2$

$69 \quad 4.2$

$77 \quad 4.5$

$114 \quad 3.3$

$84 \quad 3.7$

$32 \quad 5.5$

$97 \quad 5.3$

$54 \quad 4.3$

$70 \quad 4.3$

$108 \quad 4.0$

$86 \quad 4.1$

$25 \quad 4.6$

$70 \quad 3.8$

$36 \quad 4.8$

$61 \quad 3.7$

$59 \quad 4.1$

$76 \quad 3.2$

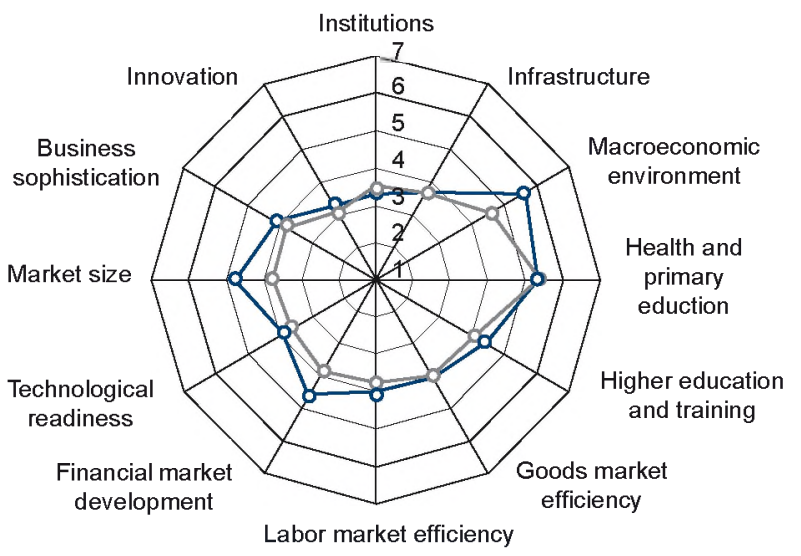

-o- Colombia $-0-$ Latin America and the Caribbean

Stage of development

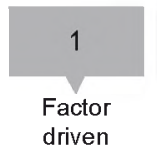

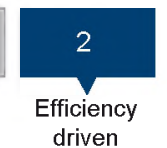

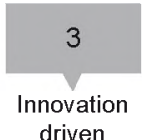

Figura 4. Desempeño general de Colombia en el Índice Global de Competitividad

Fuente: (World Economic Forum, 2015, p. 142) 


Tax rates
Corruption
Inadequate supply of infrastructure
Inefficient government bureaucracy
Complexy of tas regulations
Access to financing
Inadequately educated workforce
Crime and theft
Restrictive labor regulations
Insufficient capacity to innovate
Policy instability
Poor work ethic in labor force
Foreign currency regulations
Inflation
Poor public health
Government instability/coups

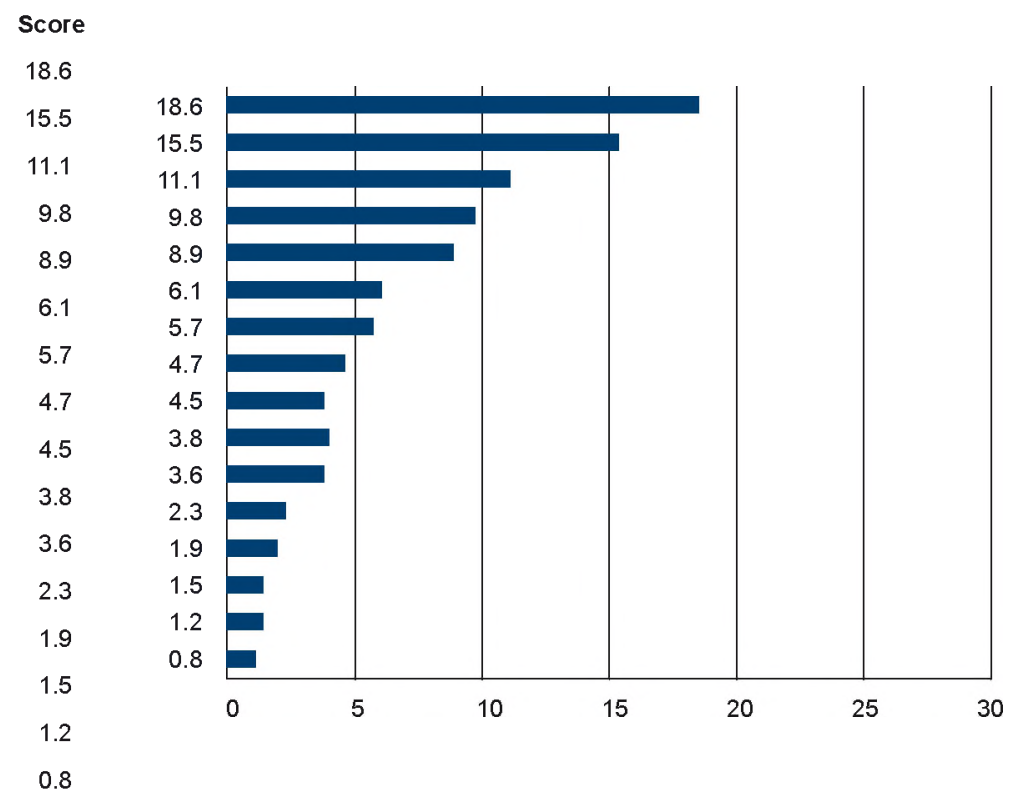

Figura 5. Resultado encuesta de los factores más problemáticos para hacer negocios

Fuente: (World Economic Forum, 2015, p. 142)

Por otra parte, en el ranking publicado en el reporte The IMD World Competitiveness Yearbook (2016), Colombia se posicionó a nivel global en el número 51 entre 60 países, y de manera desagregada se ubicó en los últimos lugares de aspectos temáticos relacionados con el desarrollo territorial. En la Figura 6 se observa como los peores resultados del país se ubican en los grupos de "government efficiency", "infranstructure", y "societal framework", en este último aspecto el país ocupa el penúltimo lugar entre 60 países -, seguidos de "education" y "scientific infranstructure".

En el último reporte Doing Business (2016), Colombia se posiciona en el puesto 54 entre 189 países. La tendencia general ha sido también positiva con respecto a las mediciones anteriores. "Colombia made the biggest improvement in the distance to frontier score in Latin-America and the Caribbean over the past 12 years. It has reformed in all areas measured by Doing Business, most notably in the areas of paying taxes and getting credit" (World Bank Group, 2015, p. 16). Pero al igual que ocurre con los rankings anteriores, se sigue observando un punto débil relacionado con el "cumplimiento de contratos", donde Colombia se posiciona en el lugar 180 entre 189 economías, con un desempeño muy bajo del 29.66/100.

Respecto a los índices de competitividad urbana existentes, ninguna de las ciudades colombianas figura en los rankings (Sobrino, 2005). Sin embargo, en el reporte Hot Spots Benchmarking Global City Competitiveness (2012) se destacan los bajos resultados que obtienen las ciudades latinoamericanas, en general, en el agregado de indicadores denominado "efectividad institucional". Se afirma, por ejemplo que "all regions have leaders and laggards in terms of competitive cities. But while most regions host at least some competitive cities, Latin America in particular performs relatively poorly across most categories, including in physical capital (its best city, Santiago, is joint 66th) and institutional effectiveness (Panama City tops the list at 53rd)" (The Economist Intelligence Unit Limited, 2012, p. 5). 

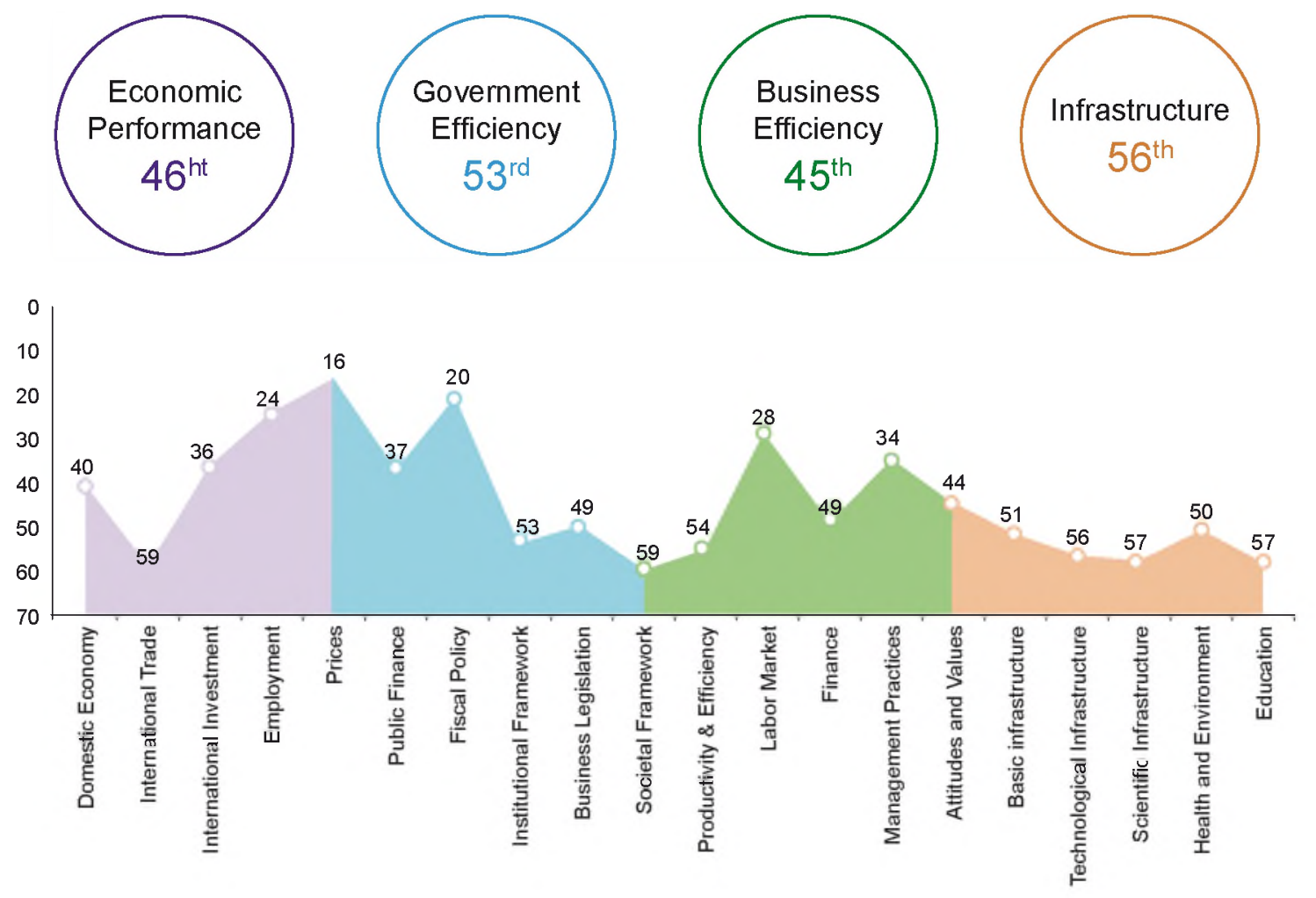

Figura 6. Desempeño general de Colombia en The IMD World Competitiveness Scoreboard 2016 Fuente: (IMD World Competitiveness Center, 2016)

\begin{tabular}{rlrr} 
& \multicolumn{1}{c}{ Declines } & WCY & wCY \\
2.5 .04 & Risk of political instability (Survey) & 2013 & 2014 \\
2.5 .02 & Personal security and private property righ & 5.98 & 4.07 \\
2.5 .09 & Equal opportunity (Survey) & 4.89 & 3.54 \\
2.5 .03 & Ageing of society (Survey) & 5.06 & 3.93 \\
4.4 .11 & Health problems (Survey) & 6.44 & 5.07 \\
2.3 .09 & Adaptability of government policy (Survey) & 4.12 & 3.27 \\
2.3 .11 & Transparency (Survey) & 4.81 & 3.82 \\
4.4 .01 & Total health expenditure (\%) & 3.29 & 2.64 \\
4.2 .07 & Connectivity (Survey) & 7.6 & 6.1 \\
4.5 .12 & Educational system (Survey) & 6.14 & 5.05 \\
4.1 .06 & Management of cities (Survey) & 4.22 & 3.49 \\
4.2 .06 & Communications technology (Survey) & 5.50 & 4.56 \\
2.5 .01 & Justice (Survey) & 6.91 & 5.75 \\
2.3 .10 & Government decisions (Survey) & 2.80 & 2.33 \\
& & 3.60 & 3.01
\end{tabular}

Figura 7. Valores más bajos evaluación de factores The IMD World Competitiveness Scoreboard 2014 Fuente: (IMD World Competitiveness Center, 2014, p. 74-75) 


\section{DISCUSIÓN}

Como se puso en evidencia anteriormente, el país aún presenta deficiencias en lo que el Foro Económico Mundial llama "requerimientos básicos" para la competitividad. En general los índices internacionales y locales muestran que el país, sus departamentos y ciudades son poco competitivos en dimensiones claves del desarrollo territorial. No se observan avances considerables al respecto, y eso es muy preocupante dado el papel que juegan en la resolución de las causas que alimentan todas las formas de violencia armada en el país.

En un evento reciente sobre Restitución de Tierras y Derechos Territoriales en la Universidad Nacional de Colombia, el director de la Unidad del Estado Colombiano encargado de esos procesos, destacaba el hecho de que buena parte del problema del conflicto armado en el país pasaba por el filtro de la falta de justicia agraria efectiva. Los indicadores de competitividad permiten confirmar que el reto más grande para elevar sus niveles en el país del pos-conflicto, no es el mejoramiento per se de las condiciones macroeconómicas, financieras, o de infraestructura física, las cuales muestran en general un comportamiento positivo y estable; sino el fortalecimiento de esas condiciones claves de ética civil que afectan con mayor fuerza las variables asociadas al desarrollo territorial. No solo se trata de más crecimiento económico o vías, sino de más justicia, de tejidos sociales fortalecidos, y de un gobierno más transparente.

En otras palabras, Colombia no avanzará sustancialmente en competitividad en la medida en que el Estado siga atendiendo de manera deficiente las condiciones que posibilitan un mayor desarrollo territorial, con el riesgo incluso de que el país retroceda los lugares que ya ha ganado en los ámbitos financieros y macro-económico.

\section{REFERENCIAS}

Affuso, A., \& Camagni, R. (2010). Territorial capital and province performance in the Latin Arch: an econometric approach. Milano. Retrieved from http://www.grupposervizioambiente.it/aisre/ pendrive2010/pendrive/Paper/affuso1.pdf

Banco Mundial. (2014). Doing Business en Colombia. Retrieved October 17, 2014, from http://espanol.doingbusiness.org/data/exploreeconomies/colombia\#enforcing-contracts

Benzaquen, J., del Carpio, L. A., Zegarra, L. A., \& Valdivia, C. A. (2010). Un índice regional de competitividad para un país. Revista Cepal, (102), 69-86. Retrieved from http://www.cepal.org/ publicaciones/xml/4/41914/rve102benzaquenetal.pdf

Buckley, P. J., Pass, C. L., \& Prescott, K. (1988). Measures of international competitiveness: A critical survey* †. Journal of Marketing Management, 4(2), 175-200. doi:10.1080/026725 $7 X .1988 .9964068$

Camagni, R. (2009). Territorial capital and regional development. In R. Capello \& P. Nijkamp (Eds.), Handbook of Regional Growth and Development Theories (p. 118-). Northampton: Edward Elgar Publishing Limited. Retrieved from http://herzog.economia.unam.mx/cedrus/descargas/Capello - HB of Regional Growth and Development Theories - 2009.pdf 
LÍNEA DE INVESTIGACIÓN: ESTRATEGIAS DE CALIDAD Y COMPETITIVIDAD

Camagni, R., Caragliu, A., \& Perucca, G. (2011). Territorial capital: relational and human capital. Milano. Retrieved from http://www.grupposervizioambiente.it/aisre/pendrive2011/pendrive/ Paper/Camagni_Caragliu_Perucca.pdf

Giaime, B. (2011). Weaving the Rural Web: The dynamics of rural development in Lunigiana. Quaderni Sismondi, (12).

IMD World Competitiveness Center. (2014). Imd world competitiveness yearbook 2014. Switzerland.

Krugman, P. (1995). Desarrollo, geografia y teoria económica. Barcelona: Antoni Bosh.

Lall, S. (2001). Competitiveness indices and developing countries: an economic evaluation of the global competitiveness report. World development,29(9), 1501-1525.

Lang, J. C. (2009). Cluster Competitiveness : The Six Negative Forces. Journal of Business and Management, 15(1), 73-94. doi:Business Source Complete, EBSCOhost, viewed 1 July 2014.

Lira, I. S. (2005). Desarrollo económico local y competitividad territorial en América Latina. Revista de La CEPAL, (85), 81-100. Retrieved from http://repositorio.cepal.org/handle/11362/11001

López Niño, D. (2015). El hexágono de la investigación. Questionar, 3(1), 148-162.

Méndez, R. (2002). Innovación y desarrollo territorial: algunos debates teóricos recientes. Revista Eure, 28(84), 63-83. Retrieved from file:///C:/Users/Inv John Ramirez/Downloads/12295916-1-SM.pdf

Organisation for Economic Co-operation and Development - OECD. (2001). OECD Territorial Outlook. Paris. Retrieved from http://www.vwl.tuwien.ac.at/hanappi/AgeSo/SecReps/Territorial_ Outlook_F.pdf

Porter, M. E. (1990). The Competitive Advantage of Nations. Harvard Business Review, 68, 73-93. doi:Article

Porter, M. E. (2000). Location, Competition, and Economic Development: Local Clusters in a Global Economy. Economic Development Quarterly, 14, 15-34. doi:10.1177/089124240001400105

Ramirez, J. F. \& Alzate, A.M. (2016). Aproximación a una valoración cienciométrica de la competitividad. Revista de Investigación, 9(1), 121-134. http: http://repository.uamerica.edu.co/handle/20.500.11839/537

Ramírez, J. C., Osorio, H., \& Isidro, R. (2007). Escalafón de la competitividad de los departamentos en Colombia. (CEPAL, Ed.) (Estudios y.). Santiago de Chile: NACIONES UNIDAS. Retrieved from http://www.cepal.org/publicaciones/xml/3/29973/lcl.2684-p.pdf

Revilla, J., Schiller, D., \& Meyer, S. (2013). Capitalising on Institutional Diversity and Complementary Resources in Cross-Border Metropolitan Regions: The Case of Electronics Firms in Hong Kong and the Pearl River Delta. In J. Klaesson, B. Johansson, \& C. Karlsson (Eds.), Me- 
tropolitan Regions. Knowledge Infrastructures on the Global Economy. Berlin, Heidelberg: Springer Berlin Heidelberg. doi:10.1007/978-3-642-32141-2

Sobrino, J. (2005). Competitividad territorial: ámbitos e indicadores de análisis. Economía, Sociedad y Territorio, (Dossier Especial), 123-183.

Sousa, S. (2011). Entrepreneurship and Institutions in European Union Regional Competitiveness. ProQuest Dissertations and Theses. George Mason University, Ann Arbor. Retrieved from http://search.proquest.com/docview/897101436?accountid=43592

The Economist Intelligence Unit Limited. (2012). Hot spots Benchmarking global city competitiveness. Retrieved from http://www.citigroup.com/citi/citiforcities/pdfs/eiu_hotspots_2012.pdf

Tóth, B. I. (2014). Territorial Capital: Theory, Empirics and Critical Remarks. European Planning Studies, 1-18. doi:10.1080/09654313.2014.928675

van der Ploeg, J. D., van Broekhuizen, R., Brunori, G., Sonnino, R., Knickel, K., Tisenkops, T., \& Oostendie, H. (2008). Towards a framework for understanding regional rural development. In J. . Van der Ploeg \& T. Marsden (Eds.), Unfolding Webs: The Dynamics of Regional Rural Development (pp. 1-28). Retrieved from http://orca.cf.ac.uk/20526/

Ventura, F., Brunori, G., Milone, P., Berti, G., Ploeg, J. D. Van Der, \& Marsden, T. (2008). The rural web: a synthesis. In J. D. Van der Ploeg \& T. Marsden (Eds.), Unfolding Webs: the dynamics of regional rural development (pp. 149-174). Retrieved from http://scholar.google.com/scho lar?hl=en\&btnG=Search\&q=intitle:The+Rural+Web+:+A+Synthesis\#0

World Bank Group. (2015). Doing Business 2016: Measuring Regulatory Quality and Efficiency. Washington D.C: The World Bank. doi:10.1596/978-1-4648-0667-4

World Economic Forum. (2015). The Global Competitiveness Report. (K. Schwab, Ed.). Geneve: World Economic Forum. 